\title{
Fiber Brewster Gratings and their applications in ultrafast fiber lasers
}

\author{
Chengbo Mou ${ }^{1, *}$, Zinan Huang ${ }^{1}$, Qianqian Huang ${ }^{1}$, Zhijun Yan ${ }^{2}$, \\ Kaiming Zhou ${ }^{3}$, Lin Zhang ${ }^{3}$, Antreas Theodosiou ${ }^{4}$, Kyriacos Kalli ${ }^{4}$ \\ ${ }^{1}$ Key Laboratory of Specialty Fiber Optics and Optical Access Networks, Shanghai Institute for Advanced \\ Communication and Data Science, Joint International Research Laboratory of Specialty Fiber Optics and \\ Advanced Communication, Shanghai University, P. R. China \\ ${ }^{2}$ School of Optical and Electronic Information, National Engineering Laboratory for Next Generation \\ Internet Access System, Huazhong University of Science and Technologies, P. R. China \\ ${ }^{3}$ Aston Institute of Photonic Technologies (AIPT), Aston University, Birmingham, United Kingdom \\ ${ }^{4}$ Photonics and Optical Sensors Research Laboratory, Cyprus University of Technology, Cyprus \\ *E-mail: moucl@shu.edu.cn
}

DOI: 10.31868/RFL2020.43-44

The rapidly developing fiber laser, with its own performance and production advantages, such as excellent beam quality, high electrical and optical efficiency, fundamental compatibility with optical systems, compact structure, and low maintenance, has gradually replaced the solid-state laser in scientific research and industrial applications. A simple, effective, and reliable passive mode-locking technique for implementing an ultrafast fiber laser remains a subject of intense interest to researchers in the laser field. For this purpose, mode-locked elements based on semiconductor nonlinear optical absorption have driven the development of novel nanomaterial saturable absorber (SA) in the past few decades. However, the high cost, complicated preparation process and low damage threshold restrict the further improvement of this kind of SA. There is another ideal way to generate ultrashort pulses is to utilize techniques based on the intrinsic Kerr nonlinearity of optical fibers. Among them, the nonlinear polarization rotation method with intra-cavity polarizing elements has been extensively used due to its high power tolerance and large modulation depth.

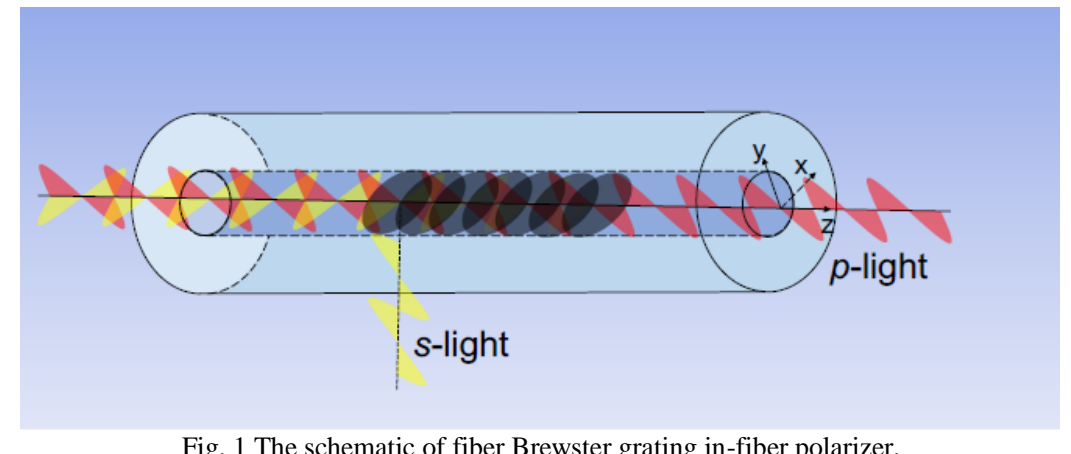

Back in $2005,45^{\circ}$ tilted fiber grating was first reported as an effective in-fiber polarizer [1] which is able to tap out the $s$-light and propagate the $p$-light based on the Brewster's law (Fig. 1). Compared with other types of in-fiber polarizers, the fiber Brewster grating own many unique advantages such as high polarizing efficiency, broadband responsivity, flexible wavelength adjustability, mechanical robustness and it can be adapted to most types of fiber. Then we successfully fabricated a UV-inscribed fiber Brewster grating with phase scanning technique and employ it as a polarizer to implement an all-fiber ultrafast laser (Fig. 2) [2]. In succession, we have used this kind of fiber Brewster gratings to achieve mode-locked erbium-doped fiber lasers with various operation regimes in $\mathrm{C}+\mathrm{L}$ wavebands, such as different pulse shaping mechanisms (conventional solitons, stretched pulses, dissipative solitons, noise-like 
pulses, etc), wavelength tunable/switchable and harmonic mode-locking. Furthermore, it also can be utilized in $1 \mu \mathrm{m}$ and $2 \mu \mathrm{m}$ mode-locked fiber lasers.
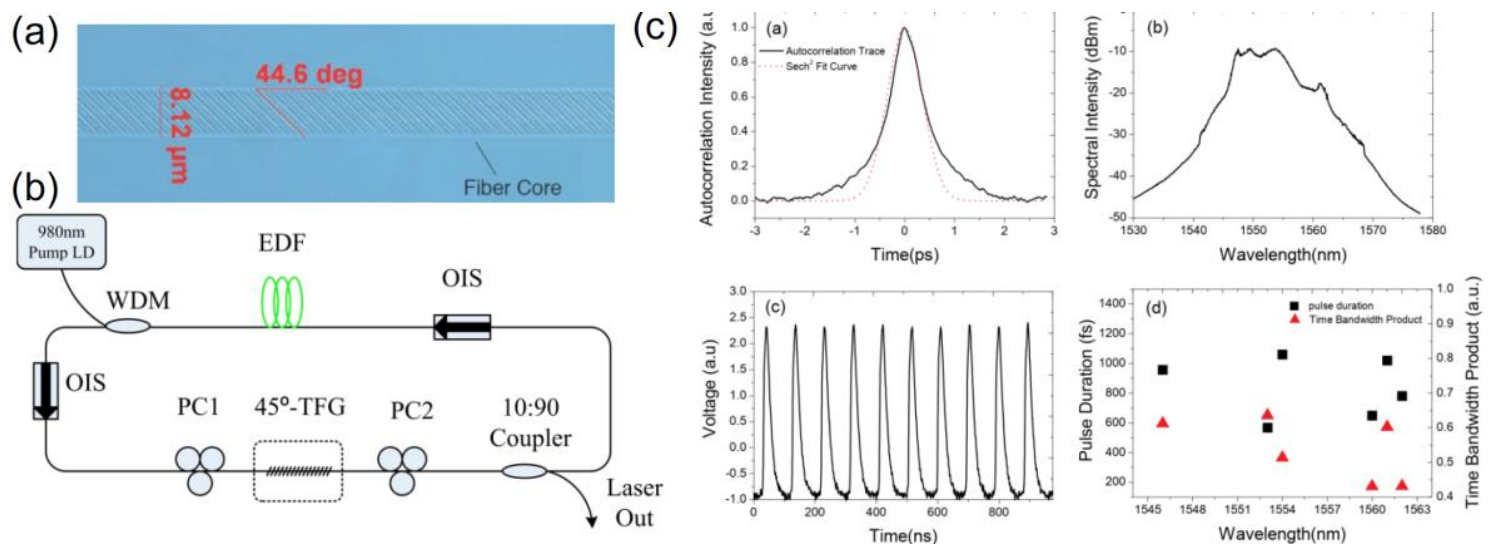

Fig. 2(a) The microscope image of the UV-inscribed fiber Brewster; the schematic (b) and measured characteristics (c) of the first all-fiber soliton mode-lockd laser based on UV-inscribed fiber Brewster grating [2].

Recently, we also have carried out research on femtosecond laser direct writing fiber Brewster grating in view of some shortcomings of UV inscription. To date, we have demonstrated that femtosecond laser inscribed fiber Brewster grating can effectively mode lock an erbium-doped fiber laser [3] and a thulium-doped fiber laser [4] in soliton regimes. In addition, the dissipation soliton and noise-like pulse output have been achieved in net-normal dispersion cavity. The femtosecond laser direct writing inscription avoids the use of expensive phase masks and photosensitivity enhancement process, which provides enormous flexibility for the design of in-fiber polarizing devices. In addition, it is not necessary to strip the fiber coating to thus maintain the mechanical strength of the grating, which is conducive to the integration and reliability of fiber lasers. Efforts are underway to further improve the performances to achieve high energy mode-locked all-fiber lasers.

(a)

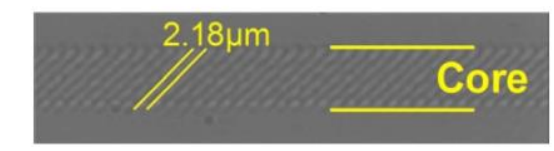

(b)

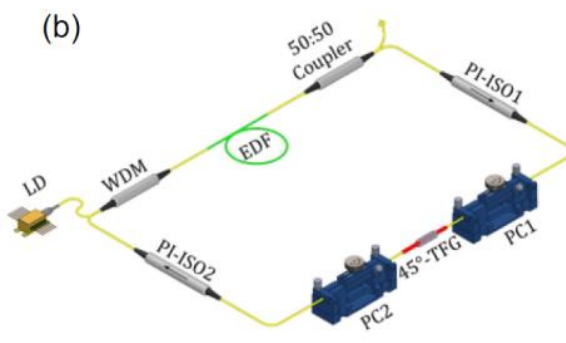

(c)
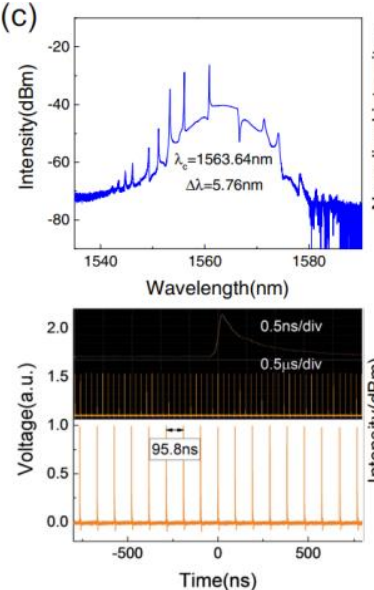
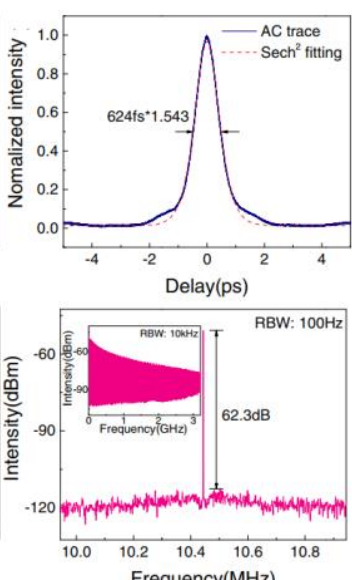

Fig. 3(a) The microscope image of the femtosecond-laser-inscribed fiber Brewster; the schematic (b) and measured characteristics (c) of the all-fiber soliton mode-locked laser based on femtosecond-laser-inscribed fiber Brewster grating [3].

The authors would like to acknowledge support from National Natural Science Foundation of China (61975107), Natural Science Foundation of Shanghai(20ZR1471500), and Ministry of Education '111' project (D20031).

\section{References}

[1] K. Zhou, G. Simpson, et al, Opt. Lett. 30, 1285-1287 (2005).

[2] C. Mou, H. Wang, B. et al, Opt. Express 18, 18906-18911 (2010).

[3] Z. Huang, Q. Huang, et al, Opt. Lett. 44, 5177-5180 (2019).

[4] N. Kanagaraj, A. Theodosiou, et al, Laser Physics Letters 16, 095104 (2019). 